\title{
Evidently epistential adverbs are argumentative indicators: A corpus-based study
}

\author{
Elena Musi ${ }^{\mathrm{a}, *}$ and Andrea Rocci ${ }^{\mathrm{b}}$ \\ ${ }^{\text {a } C e n t e r ~ f o r ~ c o m p u t a t i o n a l ~ L e a r n i n g ~ S y s t e m s, ~ C o l u m b i a ~ U n i v e r s i t y, ~} 475$ Riverside Drive, New York, NY \\ 10115, USA \\ E-mail:em3202@columbia.edu \\ b Istituto di Argomentazione, Linguistica e Semiotica, Universita della Svizzera italiana, Via G. Buffi \\ 13, 6904 Lugano, Switzerland \\ E-mail: andrea.rocci@usi.ch
}

\begin{abstract}
Argumentative indicators of discourse relations constitute crucial cues for the mining of arguments. However, a comprehensive lexicon of these linguistic devices is so far lacking due to the scarcity of corpora argumentatively annotated and the absence of an empirically validated analytic methodology. Recent studies have shown that modals, that express that things might be otherwise, and evidentials, that point to the presence of information sources, are good candidates to work as argumentative indicators. On these grounds, we propose a systematic, non-language specific corpus-based procedure to identify indicators of argumentative discourse relations. We test the design of a multi-level annotation through the analysis of the English and Italian epistential adverbs evidently and evidentemente in comparable corpora of newspaper articles. We show that the annotation guidelines achieve consistent analytical results with expert annotators. Data analysis reveals that the two adverbs work as argumentative indicator both at the structural and at the inferential level: besides pointing to the presence of premises-conclusion relations, they recurrently pattern with causal argument schemes from the effect to the cause. The Italian adverb evidentemente is less polysemous and more frequent, thus working as a more reliable indicator.
\end{abstract}

Keywords: Argumentative indicators, epistentials, multilevel annotation, newspaper articles

\section{Introduction}

Argumentation Mining aims to automatically detect and classify arguments in discourse. The way we exercise our logos to argue depends on the potentialities of our language. This awareness has led scholars in Argumentation Mining to pay attention to the linguistic underpinnings of argumentative discourse. More specifically, argumentative indicators, those "words and expressions that refer to any of the moves that are significant to the argumentative process" [42], offer crucial information: they can be exploited as features to train robust classifiers for the automatic detection of arguments. Differently from structural features (e.g. punctuation, sentence length) and n-grams commonly used in empirical works to identify argumentative components, i.e. [33,47], they are not context-specific since their indicative role is bound to their lexical semantics. Annotation projects $[34,35]$ have shown that they constitute useful cues to guide annotators' choices in the attempt of creating larger corpora argumentatively annotated. More-

\footnotetext{
*Corresponding author. E-mail: em3202@columbia.edu.
} 
over, argumentative indicators constrain the argument schemes at issue. The automatic identification of argument schemes constitutes one of the major challenges for Argument Mining.

However, the lack of an empirically-validated and context-independent lexicon of argumentative indicators undermines their relevance for Argument Mining. This is due to at least two reasons. First, argumentative indicators are discovered looking at recurrent correlations of linguistic items with argumentative features. Unfortunately, there are not enough corpora annotated as to argument structures and schemes, especially for languages other than English. This scarcity slows down the retrieval of new argumentative indicators. Second, the assessment of the predictive role played by linguistic items requires an in-depth semantic and pragmatic analysis: the indicative function played by linguistic items varies depending on their polysemy. Thus, a disambiguation process is needed. Connectives, for instance, systematically signal the presence of coherence relations and indicate argumentative relations under specific conditions [5,18]. Therefore, their relevance for Argument Mining calls for a previous mapping of discourse relations onto argumentative relations.

Besides connectives, other linguistic categories functionally close to argumentative discourse are modality and evidentiality. The former expresses that things might be otherwise: in the sentence "Mark will probably be late", the adverb probably indicates that even though unlikely, Mark could arrive on time. The latter points to sources of information supporting a statement: in the sentence "I see [Mark is climbing the stairs]", the verb see presupposes that the speaker has visual evidence to assert the proposition $p$. Modals and evidentials are highly indicative both at the structural and at the inferential levels of argumentation (see Section 2). Therefore, they promise to be relevant cues, in combination with other features, to identify and classify argumentative moves in the micro-structure of argumentation.

On these grounds, this study proposes a corpus-based methodology to investigate indicators of argumentative discourse relations [39]. The advocated procedure is exemplified through the analysis of the evidential-modal adverb evidently and its Italian counterpart evidentemente, in comparable corpora of newspaper articles. The two adverbs share the same etymological origin from the Latin $e$ - "out" + videns-entis, present participle of videre, "see". However, they are not semantically equivalent and they work slightly differently as argumentative indicators (see Section 5). Two languages are taken into account to show that our methodology is generalizable across languages. The choice of these two adverbs is motivated by their lexical semantics: differently from other evidential-modal adverbs, they impose specific constraints on the argumentative moves they indicate (see Section 3). ${ }^{1}$

\section{Related work}

Studies in Argumentation Mining have embedded discourse connectives as well as modals as features to identify the presence of argumentation and classify argumentative components, i.e. [35,36]. However, their role as indicators of specific structural and inferential configurations has so far not be taken into account. The term epistentials was coined by Faller [7] to refer to those linguistic devices which simultaneously bear a modal and an evidential function. This functional overlap generally applies to the subcategories of epistemic modality and inferential evidentiality. Recent studies at the semantics-argumentative interface have shown that a set of Italian epistential verbs work as argumentative indicators, since they constrain discourse moves at different levels of the argumentative reconstruction. In various corpusbased studies Rocci and Miecznikowski [16,28-30] show that the modal verb dovere ("must") and potere

\footnotetext{
${ }^{1}$ Although the whole paper has been the result of a continuous process of interaction between the two authors, Elena Musi is the main responsible of Sections 1, 3, 4, 5.1, while Andrea Rocci of Sections 2, 5.2, 6.
} 
("can") work as relational operators: for instance, in a sentence such as "La macchina di Giovanni non e nel parcheggio. Deve essere andato a casa" ("Giovanni's car is not in the parking lot. He must have gone home") the verb dovere links the proposition in its scope to an anaphorically available premise. This functional property is motivated by the role played by these verbs as inferential evidentials. The two verbs are, in fact, incompatible with direct and with reportative evidence:

(1) "??Giovanni deve/potrebbe essere andato a casa. Lo ho visto andare via." ("John must/could have gone home. I have seen him leaving.")

(2) "??Giovanni deve/potrebbe essere andato a casa. Me lo ha detto Laura". ("John must have gone home. Laura told me so.”).

Becker et al. [3] have shown that German modals constitute a highly frequent linguistic feature in argumentative microtexts. Moreover, different types of modal verbs seem to pattern differently with argumentative components: dynamic modals, which express ability, tend to be associated with a supporting function, while deontic modals are especially frequent with attacks of the undercut type.

Italian appearance verbs sembrare ("seem") and apparire ("appear") signal that the proposition in their scope has been inferred from a set of co-textually recoverable premises. They do not imply any directionality in the premises-conclusion relations (e.g. "Guarda le nuvole. Sembra che piovera. / Sembra che piovera. Guarda le nuvole", "Look at the clouds. It seems that it is going to rain. / It seems it is going to rain. Look at the clouds.") $[17,19,20]$. They, moreover, constrain the type of argument schemes at issue: they are both incompatible with the class of extrinsic argument schemes (see Section 4.4), such as analogical reasonings.

According to a recent corpus based study [21], English epistentials work as indicators of subjective/objective argumentative moves. More specifically, direct evidentials (e.g. see, evidence) have scope over factual propositions that play the argumentative role of basic unassailable premises: in a sentence such as "we saw what happened with the spill in Mexico" the use of the verb see specifies that the consequences of that spill were attestable by anyone through the sense of sight. The sentence, thus, works as a highly reliable premise against oil drilling. On the other hand, inferential (e.g. seem, must) and reportative evidentials (e.g. apparently) have scope on modalized propositions that play the role of local or general standpoints. Their truth is not objectively measurable but can be presented as more or less plausible depending on the status of the premises they are drawn from: in a sentence such as "He is first in the polls. He will win the elections", the likelihood of the predictions depends on the reliability of the polls.

Less attention was, instead, devoted to the analysis of epistential adverbs of certainty (e.g. obviously, patently). In sentiment analysis these adverbs are considered as indicators of degrees of commitment and belief [4,37], while their evidential function is neglected. In argumentation theory they are treated either as indicators of the degree of support that the arguments offer to the conclusion [8] or as indicators of the degree of the speaker's epistemic commitment to the standpoint $[40,41]$. In both frameworks, different expressions pertaining to epistemic certainty are considered roughly equivalent. As Freeman [11] puts it "adverbial expressions such as obviously, evidently, surely, apparently all have force", "but their cognitive meaning is sufficiently vague as to render their force the only aspect of their meaning worthy of interest. Where their cognitive meaning is clear it is synonymous with necessarily". In the same vein, Tseronis [40] argues that "there is no difference in the discourse effect that using a modal or an evidential adverb has when qualifying a standpoint". Musi and Rocci [23] have challenged this negative hypothesis in a pilot investigation. They have shown that the two epistential adverbs obviously/ovviamente and 
evidently/evidentemente exhibit both in English and Italian semantic differences. Their properties constrain in different ways the argumentative function of the propositional content they modalize as well as the way they relate to the beliefs and inferences of the arguer and of the critic. From their study it emerges that evidentementelevidently presuppose the presence of textually adjacent premises and are frequently associated with causal argument schemes. Obviously and ovviamente merely point to the presence of (inter)subjectivity. Both adverbs work at the layer of micro-argumentation: ovviamentelobviously often modalize propositions that are fairly peripheral in the overall argument, while the hidden causes uncovered in propositions modalized by evidentementelevidently function as premises to support a higher-level evaluative standpoint. On this basis, we have decided to focus our analysis on the couple of adverbs evidently/evidentemente in order to deepen and empirical validate the attested preliminary results.

\section{Data: Corpus and sample of analysis}

The proposed analysis is corpus based. The use of constructed examples has been limited to test contextual incompatibilities as a further validation of the indicative roles played by the adverbs evidently and evidentemente. We have chosen the text genre of newspaper articles for three main reasons. First, opinion articles are inherently argumentative: their communicative function is that of expressing the journalist's prise de position on an issue of public interest [12]. Second, they are bound to privilege the presence of evidentials since the management of sources of information plays a key role in the negotiation of stance [6]. Third, dealing with different topics and opinions, they guarantee the presence of a wide variety of argumentative moves. As a source, the daily newspaper New York Times and the Corriere della Sera are considered. Both newspapers were accessed through the platform LexisNexis restricting the research to articles appearing during the last year. The adverb evidentemente is present 1061 times in the Corriere della Sera, while evidently occurs 292 times in the New York Times. This difference in frequency is not limited to the text genre of newspaper articles: in the English and the Italian Web 2008 corpora, which include data from different text genres, evidently occurs 39,746 times, while evidentemente 59,945 times. As a consequence, the relevance of the Italian adverb is more prominent in an argument mining perspective.

Compared to other epistential adverbs of certainty in the same corpus, evidentemente and evidently are not the most frequent (see Table 1).

Table 1

Frequency of epistentials of certainty in the last year articles from the New York Times and the Corriere della Sera

\begin{tabular}{llc}
\hline Language & Epistentials adverbs & Number of occurrences \\
\hline English & clearly & 7458 \\
English & obviously & 2014 \\
English & evidently & 292 \\
English & patently & 66 \\
English & manifestly & 22 \\
Italian & ovviamente & 2684 \\
Italian & evidentemente & 1061 \\
Italian & chiaramente & 774 \\
Italian & palesemente & 128 \\
Italian & manifestamente & 12 \\
\hline
\end{tabular}


Both in English and in Italian the adverbs obviously/ovviamente, are more frequently attested than $\mathrm{ev}$ idently/evidentemente. Moreover, in English also the adverb clearly is far more frequent than evidently. We have chosen to focus on evidently/evidentemente since they seem more informative as argumentative indicators. The adverbs obviously/ovviamente, as shown in [23], constrain argumentative discourse in a looser way than evidently/evidentemente: their argumentative role is to indicate that the modalized proposition is presented as inferable by the addressee, and frequently not inferred by the speaker (who knows it from direct experience). In other words, the premises from which the inference is drawn are always intersubjective, namely accessible to the hearer. However, the premises are in circa half of the occurrences not textually recoverable; when textually expressed they tend to be not textually adjacent [23]. Turning to the analysis of argument schemes, it has emerged that obviously/ovviamente are compatible with both intrinsic and extrinsic argument schemes, though having a preference for the former. Therefore, the predictive function of this couple of arguments for the retrieval of specific argumentative move is quite vague.

The adverb clearly works frequently as a manner (e.g. "The teacher explained the matter clearly") rather than as a sentence adverb (e.g. "He has clearly already won the elections", "Ha chiaramente gia vinto le elezioni"). When working as a manner adverb, it does not bear an epistential function and lacks any indicative value for argumentation.

In order to obtain a comparable sample of analysis a sample of 50 articles per language, each containing at least one occurrence of the adverbs, has been selected starting from the most recent ones in chronological order. The adverb evidently is attested in 106 occurrences/89,242 tokens, while the adverb evidentemente occurs 75 times/60,588 tokens.

\section{Methods: Multi-level annotation}

The annotation has been conducted using UAM Corpus Tool (ver. 3.3e). It was undergone by the two authors, who are also responsible of the guidelines' design. The aim of the annotation process was not, in fact, that of building feasible guidelines to be used in crowd-sourced experiments: the devised semantic and argumentative annotation principles call for highly expert annotators. The goal was, instead, that of offering a systematic methodology to assess the indicative role played by epistentials. We hope that this procedure will be used by other expert annotators to investigate argumentative indicators. The annotation schemes as well as the annotated corpus are publicly available: https://github.com/ musielena/epistentialsmultilevelannotation. The designed annotation encompasses four main layers (see Table 2).

The order of the annotation layers reflects their inter-dependence. Epistentials have by definition scope over a proposition: thus, the semantics layer applies to every-occurrence. The presence of speaker's/hearer's inference is, for instance, not at issue when the proposition in the adverbs' scope is factual and directly accessible to the speakers. The syntactic level applies only to cases where the proposition is inferred and the premises are expressed; the types of argument schemes at issue can be assessed only when premises are accessible. We have calculated the inter-annotator agreement (using

Table 2

Levels of annotation

\begin{tabular}{llll}
\hline Semantics & (Inter)subjectivity & Syntax/Discourse structure & Argumentation \\
\hline $\begin{array}{l}\text { Proposition types in the } \\
\text { adverbs' scope }\end{array}$ & $\begin{array}{l}\text { Speaker/hearer inference and } \\
\text { heteroglossic functions }\end{array}$ & $\begin{array}{l}\text { Synatctic/anaphoric } \\
\text { manifestations of premises }\end{array}$ & Types of argument schemes \\
\hline
\end{tabular}




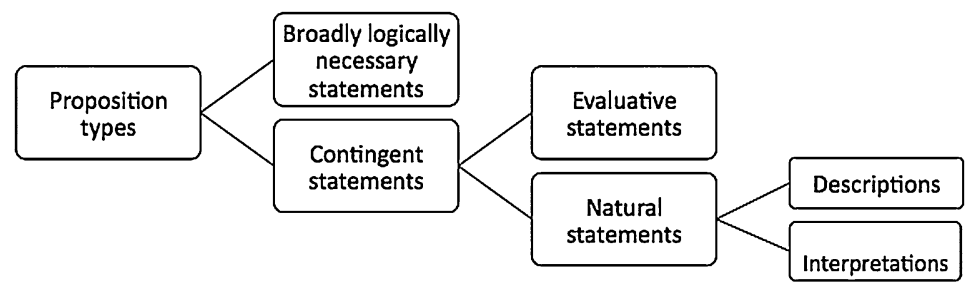

Fig. 1. Taxonomy of semantic types of propositions.

Kohen's kappa) for the annotation levels which require a high level of interpretation - semantic type of proposition in the adverb's scope and argument schemes. Occurrences leading to divergent annotations are likely to be those constructions where evidently and evidentemente do not constitute decisive pointers of argumentative moves. Each layer of analysis is meant to be generalizable for the study of other argumentative indicators.

\subsection{Semantic level}

This level addresses the semantic types of proposition over which the adverbs have scope. As shown by recent studies, proposition types correlate with specific argumentative components [2] and constitute useful knowledge for the automatic classification of argument schemes [15]. The adopted taxonomy roughly follows that proposed by Freeman [9], based on the dichotomy between broadly logically necessary statements, whose truth/falsity is logically determinate (e.g. "One physical object cannot be in two places at the same time") and indeterminate or contingent statements (see Fig. 1).

Broadly logical necessary statements occur rarely in everyday prose and, when they occur, they hardly ever occur with epistentials. This is because they do not need evidence to be known: their truth does not depend on external contingent facts and is graspable through our common intuitions. Among contingent statements a distinction is made between evaluative and non evaluative, natural propositions:

- Evaluations: they contain an axiological predicate - i.e. "Cio ha esiti evidentemente paradossali", "That has evidently paradoxical outcomes", source Corriere della Sera.

- Natural statements-interpretations: they express presumptions about explanatory relations between states of affairs, such as causal relations - i.e."After a year of exceptionally good sales years, the market is evidently saturated"; they are compatible both with direct and inferential evidentials.

- Natural statements-descriptions: they encode facts in the actual word potentially experienceable through perception - i.e. "The table in front of me is made of wood" - or internal states available through introspection (i.e. "I am feeling very tired tonight").

Among interpretations predictions (i.e. "Evidently, he will be joining the attack", source New York Times) have been singled out since preferably associated with certain subtypes of argument schemes: they, for example, favor the presence of relations from the cause to the effect rather than from the effect to the cause. This level of analysis is exportable to the analysis of any potential argumentative indicator having a propositional scope. 


\subsection{Intersubjective level}

This level applies both to sententially and lexically-driven aspects. As to sententially-driven aspects, the proposition can be presented as the result of an inference of the speaker and/or as inferable by the hearer. When presenting a proposition as inferred, speakers index their own thought processes; this has two argumentatively relevant implications: the hearer may be invited to follow the same path of reasonings [26]; at the same time the hearer is warned that the proposition is a debatable standpoint. Moreover, inferred propositions hints to the available premises excluding direct access to the encoded situation as well as hearsay or report as sources of information.

The reasoning path of the speaker, however, can be followed by the hearer only when the proposition is also inferable by the hearer. Presenting a proposition as inferable by the hearer means pointing to the availability of contextual or co-textual premises. In such cases the premises constitute non-subjective evidence and express intersubjectivity as intended by Nuyts [24]. According to the scholar, the dimension of subjectivity does not rely on the quality of the speaker's evidence (mathematically measurable or vague) in support of an epistemic evaluation, but rather on the interactive terms of the question whether the evidence is available only to the speaker or more widely known. The presence of (inter)subjective premises enhances persuasion.

Lexically driven aspects deal with the heteroglossic functions played by the adverbs, as intended in Appraisal Theory. Appraisal Theory [44,45] is a framework drawing from the main principles of Systemic Functional Linguistics [13]. Its main goal is to investigate and explain how stances are taken and managed in interaction (heteroglossia) looking at how language is used. Appraisal Theory differs from truth-functional semantic theories: according to Appraisal Theory the communicative function of the verb may in a sentence such as "Sally may be wrong" is not that of mitigating the speaker's commitment to the truth of the proposition "Sally is wrong", but of recognizing the presence of other conflicting beliefs (i.e. "Sally is right", "Sally is partially wrong").

According to the Appraisal framework heteroglossic strategies are grouped under the label of engagement, which refers to all the linguistic constructions which allow speakers to express and negotiate their positions engaging in multiple voice interactions. As shown by Vandenbergen et al. [32], certainty adverbs are privileged linguistic strategies to indicate the heteroglossic function engagement. Following White [45], the typology of heteroglossic functions is the following:

- Concurrence: it expresses agreement/sharedness of knowledge or points of view on the part of the speaker with the hearer - examples of typical markers are obviously, of course;

- Pronouncement: it expresses authorial emphases though interventions where the speaker's subjectivity is highlighted - typical markers are certainly, definitely, clearly;

- Endorsement: it gives voice to external voices' opinions which are construed by the speaker as correct or in any case highly warrantable - examples of typical markers are demonstrate, show, indeed;

- Concession: it expresses the speaker's countering of a point of view by first agreeing with some aspects of it - examples of constructions with a concessive value are I agree with you/sure,... but/however;

- Counterexpectancy: it expresses the speaker's rejection of a reasonable viewpoint drawn from expectations - typical markers are contrastive connectives such as but and instead.

Knowledge about linguistic resources to express Engagement is crucial for the analysis of argumentative moves for at least two reasons. First, heteroglossic strategies work as rhetorical strategies in the 
sense that they allow the speaker to position his point of view in alignment or in disalignment with other viewpoints. Second, this negotiation of stances is at the core both of the confrontation stage and the opening stages of a critical discussion [41] where participants respectively establish their disagreement space as well as their common beliefs. According to Appraisal Theory epistentials inherently work as heteroglossic strategies: modals point to the presence of a set of alternatives opening up a dialogic space to account for any of them; evidentials point to the presence of sources of information which are communicatively relevant only if considered in an interpersonal perspective. Therefore, they are likely to prompt the recognition of argumentative moves of support or attack of opinions in the speaker/hearer interaction.

\subsection{Syntactic/discourse structure level}

This level applies to the occurrences in which the proposition in the adverbs' scope is presented as inferred by the speaker and/or inferable by the hearer. It addresses whether the premises at the basis of the inferred proposition are textually manifested and what is their position among the following options:

- intraclausal: premises and inferred proposition belong to the same clause, i.e. "the level of their inability to communicate reveals that the kinky predilections [hat each of them has evidently been hiding from one other", source New York Times;

- intrasentential: premises and inferred proposition belong to the same sentence, i.e., "Cio ha esiti evidentemente paradossali, come quello di considerare Cristoforo Colombo alla stregua di Adolf Hitler", "That has evidently paradoxical outcomes, such as that of considering Christopher Columbus like Adolf Hitler", source Corriere della Sera;

- intersentential: premises and inferred proposition appear in adjacent sentences. The reader is invited to infer a short distance discourse relation between the adjacent sentences, i.e., "II is time to show off some samurai spirit', her husband boasts. Evidently he will be joining the attack", source New York Times;

- multiple manifestations: premises are scattered throughout the article showing multiple manifestations; this may involve long distance discourse relations and resumption of topics.

The textual explicitness as well as the position of the premises are informative about the indicative potential of the adverbs in signaling the presence of argumentative discourse relations.

\subsection{Argumentative level}

This level refers to the argument scheme that is activated in the inferential step from the premises to the proposition modalized by the adverbs. It, therefore, applies to the occurrences in which the premises in support of the truth of the proposition in the adverbs' scope are textually expressed or easily recoverable from common ground knowledge. The adopted taxonomy of argument schemes is that proposed in the Argumentum Model of Topics (AMT) framework [27]. Compared to other taxonomies of argument schemes ([25,43]) it offers at least two advantages for annotation purposes: it is hierarchical, thus avoiding a proliferation of argument scheme. Moreover, it is based on frame semantic, non-context sensitive criteria (see Fig. 2).

At the higher level, a tripartite distinction is made among:

- Intrinsic argument schemes: the state of affairs expressed by the premise and that expressed by the claim belong to a unitary frame, either within the same possible world (e.g. parts and whole) or in causally accessible possible worlds (e.g. material cause); 
- Extrinsic argument schemes: the state of affairs expressed by the premise and that expressed by the claim belong to separate, independent or alternative, frames that are compared or contrasted;

- Complex argument schemes: they have a pragmatic rather than a semantic-ontological connection with the standpoint.

At the middle level, the semantic and pragmatic connections linking premises and claims are further subclassified. We have adopted the operational definitions provided in the guidelines proposed by Musi et al. [22]: ${ }^{2}$

- Definitional: the claim expresses a categorization (x is a y) and the premise expresses properties that allow the categorization.

- Mereological: either (i) entities in the premises constitute parts of a whole expressed in the claim (or vice versa) or (ii) the events expressed in the premises are exemplary cases or a good number of cases from which a generalization or a prediction is drawn (induction).

- Causal: the premises and the conclusions are connected by causal relations.

- Analogy: the state of affairs expressed in the premise and that expressed in the claim encode situations which have occurred in different spatio-temporal circumstances but that are similar in some respects.

- Opposition: the state of affairs expressed in the premise (or one of its entities) is opposite with respect to the state of affairs expressed in the claim (or one of its entities); this means that the occurrence of one of the two excludes the occurrence of the other one.

- Practical evaluation: the state of affairs expressed in the premise is an evaluation, namely a judgment about something being 'good' or 'bad'. The claim expresses a recommendation/an advice about stopping/continuing/setting up an action.

- Alternatives: the realization of the state of affairs expressed in the claim is valid since every other possible alternative to it, expressed in the premise, is excluded.

- Authority: the premise is a discourse uttered by a person who is highly reliable/is an expert/is an authority in the context of discussion and the propositional content of the claim coincides with the content of that discourse.

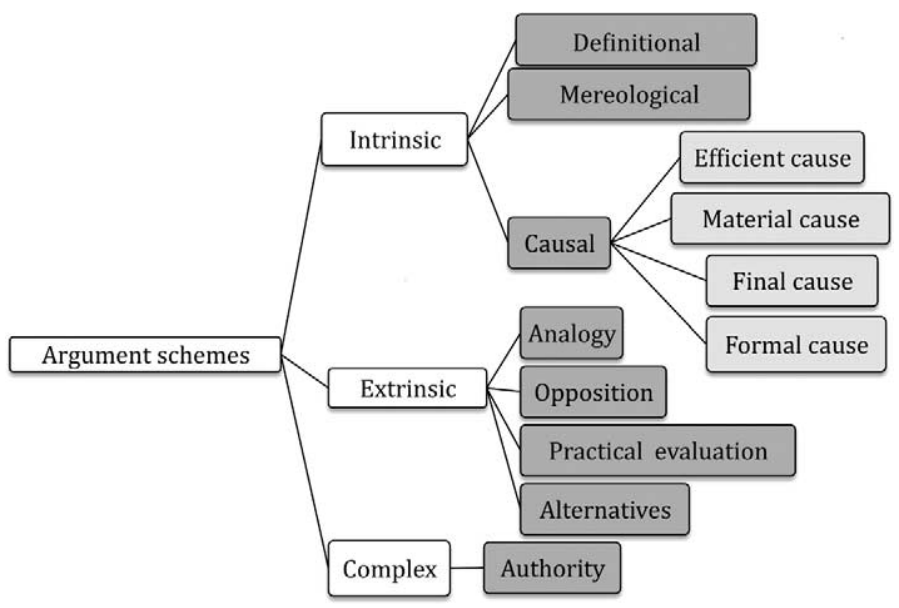

Fig. 2. AMT typology of argument schemes.

\footnotetext{
${ }^{2}$ For a complete taxonomy of argument schemes as intended in the Argumentum Model of Topics see [31].
} 
At the lower level, causal argument schemes are distinguished depending on the four Aristotelian cause types. Those cases where premises are not accessible have been annotated with the label no discernible to account for the impossibility of identifying an argument scheme.

\section{Analysis of the results}

\subsection{Evidently}

The annotation of the semantic type of proposition has received perfect agreement. The English adverb evidently has in our sample mainly scope over propositions of the interpretative type ( 75 occurrences), followed by evaluations and descriptions, according to the following distribution (see Fig. 3).

The compatibility of the adverb with descriptive propositions could prima facie suggest that the adverb is compatible with direct evidentiality [46]. Direct evidentiality is, in fact, the most suitable evidential mode to present factual state of affairs since it points to direct experience (and testimony) as an information source. However, even in those cases, the presence of evidently entails that the speaker has not directly witnessed the state of affairs encoded by the embedded proposition:

(1) "On the screen was a man wearing a cowboy hat and a shiny shirt, strumming a guitar and, evidently, singing a song. I say evidently because the sound was off", source New York Times.

As explained by the author himself, the use of the adverb is justified by the inaccessibility for the speaker of the singing event in its entirety: the speaker infers from the lip movements that the cowboy is singing, even though he cannot hear the song. The proposition is presented as factual due to the high reliability of the perceptual source of information. In such a context, evidently could be replaced by the epistential adverb apparently, but not by clearly, even though both the adverbs share with evidently an etymological reference to vision (apparently < Latin apparent-em < apparere, "to come into sight"; clearly < Latin clarus, "full of light"). The sentence "I say clearly because the sound was off" would sound inappropriate. Differently from "evidently" and "apparently", "clearly" tends to have scope over events directly experienced by the speaker, i.e. "It is 11.00 p.m. and he was supposed to be here at 10.00 p.m. He is clearly/*evidently/*apparently late". On the other hand, the adverbs evidently/apparently work as inferential evidentials, incompatible with direct evidentiality.

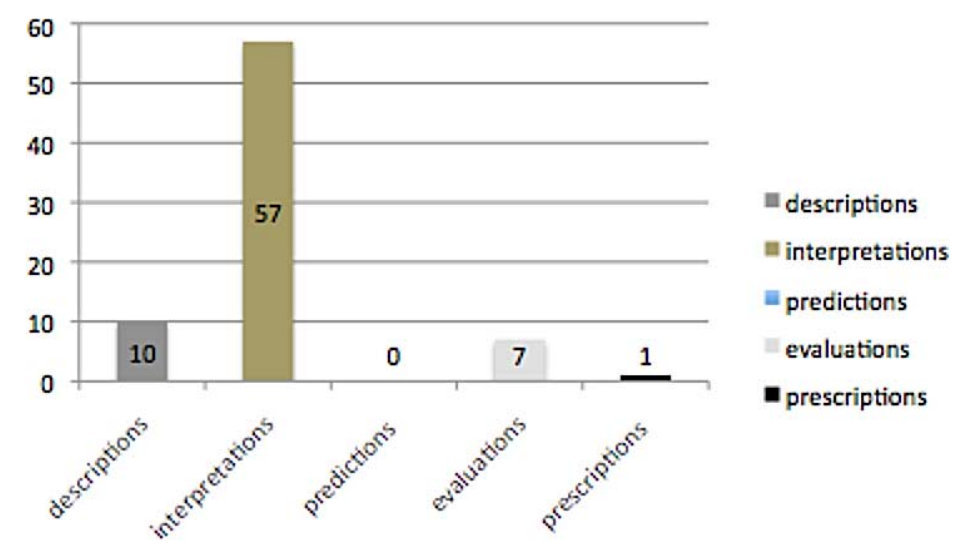

Fig. 3. Distribution of semantic types of propositions in evidently's scope. 
In the other 3 occurrences labeled as descriptive the adverb indicates experiential inference [1] since based on data perceptually available:

(2) "Inside the showroom late Wednesday, several men in gray suits studied the now empty case, murmuring and pointing to the wooden trim along its bottom edge where the lock had evidently been sprung", source New York Times

In those cases the modal value of the adverb is backgrounded, while its evidential meaning, foregrounded, corresponds to "in a way that was easy to see" [32]. In other words, when indicating experiential inference, the adverb is reminiscent of its original value as a manner adverb [38]. Such a meaning is not anymore transparent with the adverb apparently, which would sound inappropriate in these contexts. Besides inference as an information source, evidently can indicate that the proposition in its scope is true according to what somebody other than the speaker/other people in general have said:

(3) "Mr. Birkhofer described the disaster as a 'design-basis accident', indicating that safety systems had evidently proven insufficient", source New York Times;

(4) "There were reports that Mr. Netanyahu met with newspaper editors today, evidently to urge restraint in covering the affair", source New York Times.

Therefore, evidently neutralizes the distinction between inference and hearsay or report as information sources, working as a marker of indirect evidentiality. It has to be noticed that while evidently can be replaced with apparently in example 3., it would sound less suitable in example 2. The adverb apparently is used to indicate hearsay but it is generally not attested when the information source is a third person. Occurrences where the adverb assumes an hearsay or a reportative function emerge from the annotation as those where the proposition in the verb's scope is not the result of the speaker's inference and, at the same time, it is not inferable by the hearer. They all encode interpretations and they include the 4 occurrences in which the interpretation is of the predictive type. These tendencies highlight the modal flavor of the adverb which indicates high speaker's commitment: states of affairs projected in the future are the less suitable to be presented as certain. In every occurrence where evidently indicates inference there is also inferability on the part of the hearer: when the premises are textually expressed (57 occurrences) they tend to be intrasential or in a sentence textually adjacent, allowing the hearer to easily reconstruct the speaker's inferential path. When the premises are left implicit (48 occurrences), they contain information which belongs to encyclopedic knowledge, thus recoverable even if not expressed:

(5) "But not last Thursday, when the front of the plane was filled with passengers who had evidently actually paid the first-class fare, leaving no seats available for upgrades", source New York Times.

The premise which allows the speaker to conclude that the passengers sit in the front of the plane have all paid the upgrade is commonsensical information about the plausible policies of the airline (i.e. "Passengers who paid the actual first-class fare have priority over passengers that just paid the upgrade"). In such occurrences the modal value of the adverb, which indicates epistemic certainty, is more prominent than its evidential function.

Turning to heteroglossic aspects, the distribution of the adverb's functions seem to pattern with its evidential and modal values: when the evidential value of the adverb is backgrounded in favor of its modal value, evidently expresses concurrence since the truth of the embedded proposition is presented as apparent to everyone. This is the case not only when the premises are left implicit, but also when they are basic unassailable premises [10] whose factuality can be verified by the hearer: 
(6) “There are parallels to the playwright's family in some of the play's characters, but it is not, evidently, a genuinely autobiographical work; her father was a landscape painter and not the tyrannical and closed-minded businessman that the title character is", source New York Times.

Besides concurrence, which appears to be the most frequent rhetorical function in the considered sample (56 occurrences), the adverb is also employed in 39 occurrences to express pronouncement. This happens generally where its inferential evidential function is more prominent and where the premises, though textually expressed, are non basic premises:

(7) "Can she really take pleasure in plumping for an autofill ideology that's so widely shared? Evidently she can. The book, which on one level is a chronological account of Winthrop's attempt to build an exemplary "city upon a hill," hums along with chipper personal details and relaxed talk-show-guest banter", source New York Times.

The conclusion drawn by the speaker about the ideological intents' of the author of the book is based on his personal evaluations of the writer's narrative style. Therefore, it could potentially not meet other readers' opinions. In such cases, the presence of textually expressed premises assumes the rhetorical function of presenting the speaker's claim as grounded, and hence, reasonable. This intent is confirmed in example 6. by the presence of a rhetorical question which "contributes to enhancing the persuasive force of the implicated assertion" [14]. When it indicates hearsay or report, evidently matches with endorsement: the journalist does not take epistemic distance from the truth of the propositions in the adverb's scope attributing them to somebody else, but he endorses them. It has to be remarked that, differently from evidential verbs such as demonstrate or show, the adverb does not lexically presuppose endorsement, but this rhetorical function is bound to the specific text genre: the journalist gives voice to experts or public characters whose claims mainly contribute as premises to frame the journalist's standpoint. An exception is the following context, where the journalist's dis-alignment with the public announcement is made explicit:

(8) "The need for, and usefulness of, surveillance was given fresh significance after the bombings in London last week, and in particular with the announcement on Tuesday that cameras had evidently helped to identify likely suspects, including at least one presumed suicide bomber. But while cameras were evidently critical in this investigative breakthrough, the London attack also underscored their limitations: the cameras, of course, could not and did not prevent the explosions", source New York Times.

In its first occurrence evidently assumes a typical hearsay value giving voice to the media opinion about the usefulness played by cameras as surveillance means in case of terrorist attacks. In the second occurrence the adverb is part of a concessive construction through which the speaker expresses partial disagreement with the media: although he concedes that cameras constitute effective means to find the responsible of the terrorist attacks, he points out that their advocated usefulness is undermined by their proven incapability of preventing the attacks themselves.

The annotation of the middle level argument schemes has received substantial agreement (overall agreement $k=0.76$ ), showing that the proposed guidelines lead to similar annotation results with expert annotators. As visualized in Fig. 4, the major aspect of disagreement concerns the label no discernible.

From the qualitative analysis of cases of disagreement between the two annotators, two different tendencies emerge: the first annotator has the tendency to not reconstruct the argument scheme at work where the premises are textually implicit; the other tries to supply the implicit premises where inferable with reasonable likelihood. From both annotations it emerges that evidently mainly selects intrinsic 

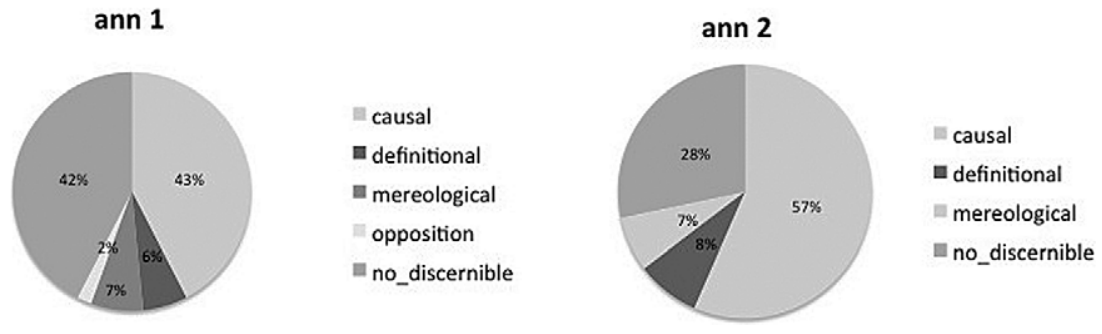

Fig. 4. Distribution of argument schemes with evidently.

argument schemes, with a clear preference for those of the efficient-causal type. Moreover, it seems to impose constraints on the direction of causality which recurrently proceeds from the effect to the cause and, more specifically, from human observable behaviors to not observable intentions, values or internal states:

(9) “The first Beirut album, Gulag Orkestar (Ba Da Bing!), was released earlier this year, and it's not an album anyone could have been expecting. Mr. Condon, who grew up in New Mexico but now lives in Brooklyn, is evidently obsessed with Balkan Gypsy music. (In the album's liner notes, he 'thanks all Balkan brass bands'.) That explains the clattering arrangements, and maybe also the melancholy melodies", source New York Times.

In example 9. the journalist tries to explain the peculiarities of the new Beirut's album attributing to Mr. Condon's a new obsession for Balkan music. He presents this interpretation of the singer's internal state as an inference drawn from information contained in the following two propositions. On the one hand, the expressive speech act contained in the liner notes constitutes a symptom of Mr' Condon's general attitude. The use of the quantifier all well matches with the intensity of sentiment that characterizes an obsession: the singer is not thankful to a specific band, but to the overall music genre as a source of inspiration. On the other hand, through the use of the verb explain, the journalist abductively presents the unexpected quality of the arrangements and the melodies as effects of the artist's fixation. This tendency for signaling an inferential path starting from perceivable human behavior is accessible also when the adverb signals other intrinsic argument schemes:

(10) "The problem of women being invasively patted down and made to remove clothing at checkpoints has eased, but it evidently hasn't disappeared. Alexandra Mack, a workplace anthropologist from Connecticut, said she attended a business conference in Santa Fe, N.M., in early April and brought along her 6-month-old son. On the return trip, with infant in arms at the Albuquerque airport, she reports: "I was dressed in slacks, a T-shirt and a long-sleeve cotton button-down. I was told to remove my long-sleeve shirt since it was outerwear. I saw no one else asked to remove similar articles of clothing. At that point, undressing was physically impossible while I was also holding a small child.", source New York Times.

In example 10. the premise from which the journalist's prise de position is drawn is the personal experience lived by Alexandra Mack who had to suffer from the security's unreasonable request to remove her long sleeves shirt. This singular behavior is construed by the journalist as prototypical example that makes him draw generalizations about the checkpoints sexist habits. The representativity of the cited examples is stressed by the fact that the Alexandra Mack is described as the specimen of a respectable and non-suspected person. 


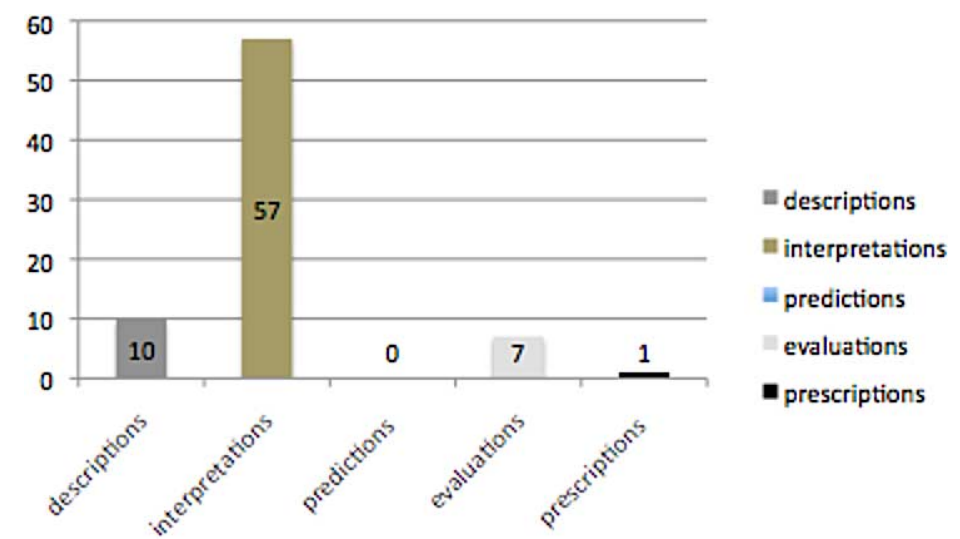

Fig. 5. Distribution of semantic types of propositions in evidentemente's scope.

\subsection{Evidentemente}

Like for the adverb evidently, the annotation of the semantic types of proposition in the scope of evidentemente has reached perfect agreement between the two annotators. However, their distribution is not equivalent to that attested with the English counterpart (see Fig. 5).

From the comparison between Figs 3 and 5, it appears that evidentemente introduces descriptions (i.e. "E a fatica (evidentemente provato) ha ripercorso e ribadito punto per punto la sua versione", "And putting effort (evidently in difficulty) he retraced and reiterated in detail his version") less frequently than evidently. In contexts where experiential evidentiality is at stake, such as example 2., the Italian adverb palesemente ("patently") would fit better than evidentemente ("evidently"). This annotation result well patterns with the different syntactic behavior of the two adverbs: taking a random sample of 100 newspaper articles from the Corriere della Sera and the New York Times it appears that evidentemente is attested in sentence initial position (21/116 occurrences) more frequently than evidently (26/193 occurrences). This evidence suggests that evidentemente works more frequently than evidently as a sentence rather than a manner adverb, having that propositional scope which is necessary for a modal-evidential value to arise. This tendency could suggest that the Italian adverb is more advanced in the process grammaticalization and subjectification encountered by Traugott [38] in her diachronic analysis of evidently. An argument in favor of this tentative explanation is that the adverb appears to be more frequently associated to evaluations, which pinpoint the speaker's subjectivity.

As to evidential values, the adverb differs from evidently in that it cannot indicate hearsay: if we had to translate in Italian example 3. we would not use evidentemente in correspondence of evidently, but the locution a quanto pare ("as they say") or the verb sembra ("seem") ("Ci sono state segnalazioni che Netanyahu ha incontrato i direttori dei giornali di oggi, a quanto pare/sembra/??evidentemente per sollecitare moderazione nella copertura della vicenda"). It is however, compatible with inferential readings where the sources of information are discourses uttered by third parties:

(11) "Come sosteneva Vilfredo Pareto nel 1906 la fondazione della economia politica e evidentemente la psicologia ed un giorno si potranno dedurre le sue leggi come quelle delle altre scienze sociali da essa."

"As claimed by Vilfredo Pareto in 1906, psychology is evidently the foundation of political economy and one day you will be able to deduce its laws from it, as it is the case for other social sciences", source Corriere della Sera. 
In examples such as example 11., the rhetorical function displayed by evidentemente is genuine endorsement. Differently from cases of hearsay, in which the journalist does not directly commit to the embedded proposition (cf. example 7.), in these cases it would be difficult to conceive his later distancing from Pareto's point of view. Besides the management of endorsement, the other main heteroglossic functions are those of concurrence and pronouncement. Differently from evidently, evidentemente shows a preference for the latter in line with its more prominent inferential meaning (40/75 occurrences). As a further proof of this semantic difference between the two adverbs, evidentemente is more frequently paired with textually expressed premises ( $80 \%$ vs. $56 \%$ with evidently).

Turning to the annotation of argument schemes, the interannotator agreement is substantial $(k=0.71)$. The distribution of the argument schemes as conceived by the two annotators is visualized in Fig. 6 .

The role of evidentemente as a stronger indicator of premises-conclusion relations than evidently is confirmed by the presence of less occurrences where an argument scheme is not discernible. Morever, evidentemente seems to be more specialized than evidently in indicating causal relations of the efficient type with an effect-cause direction:

(12) "Il guaio pero e che nelle settimane scorse sono circolati studi e tabelle di segno divergente a dimostrazione evidentemente di difficolta / ritardi a monte, nella registrazione degli input."

"The trouble, however, is that in recent weeks studies and divergent-signed tables have circulated, evidently demonstrating upstream difficulties / delays in registering the input", source Corriere della Sera.

In example 12., the relation between the premises and the inferred proposition over which evidentemente has scope is explicitly marked by the locution "a dimostrazione" which licenses the inferential rule "if the effect is the case, the cause is the case". In this occurrence, where the evidential function is already signaled by locution, the meaning of evidentemente is prevalently that of expressing modal certainty.

It has to be noticed that, though presupposing premises in the vicinity, evidentemente does not impose constraints of the premises' positions which can appear both before o after the inferred proposition:

(13) "Il dossier elenca i soldi stanziati, gli interventi effettuati, il nome dei progettisti, le ditte incaricate. Indica anche l'effettuazione dei collaudi per la convalida di quanto era stato fatto. Interventi per una spesa ingente, che evidentemente non erano stati svolti adeguatamente, visto che alcuni edifici sono stati distrutti dal sisma di sei giorni fa e altri risultano gravemente lesionati"

"The file lists the money allocated, the action taken, the name of the designers, the companies entrusted. It also indicates the performance of the tests for validation of what had been done. These actions have caused major expenses and were evidently not carried out properly, as some buildings were destroyed by the earthquake six days ago and others are seriously injured", source Corriere della Sera.
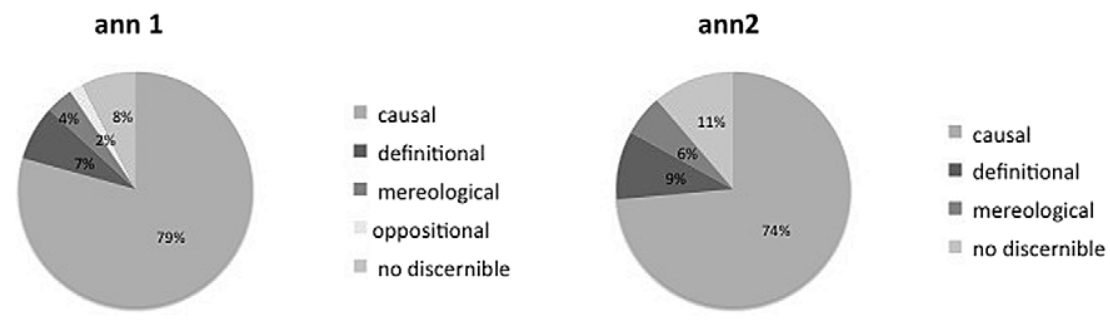

Fig. 6. Distribution of semantic types of propositions in evidentemente's scope. 
In example 13. the high degree of epistemic commitment conveyed by the adverb is justified by the factual evidence of the sad effects that which are incompatible with effective previous infrastractural interventions.

\section{Conclusion}

This study contributes to Argument Mining proposing a methodology for the uncovering of argumentative indicators. Argumentative indicators, such as epistentials, constitute useful features for classification experiments aimed to mine arguments. Although highly indicative, modals and evidentials are too infrequent to automatically map the argumentative configuration of text. However, they promise to improve precision since, compared to shallow and structural features, their indicative role depends on their semantic and is less contextual variable.

Compared to previous analyses, the devised procedure is systematic, non language specific and reproducible. It is based on a multilevel annotation which encompasses semantic, (inter)subjective, syntactic/discourse structural and argumentative features. Each of these levels is, in fact, highly significant in an argument mining perspective: semantic types of proposition are associated to specific argumentative components (claims, premises); the management of speakers'/hearers' inferences guides the unfolding of arguments; argument schemes allow us to detect the displayed reasonings that may lead to persuasion. As a proof of concept, we have applied our methodology to the English and Italian epistential adverbs evidentementelevidently in comparable corpora of newspaper articles. We have calculated the interannotator agreement for the identification of the semantic types of proposition in the adverbs' scope and for the argument schemes. These two tasks require a deep interpretation and are, thus, more likely to give rise to divergences. They respectively received perfect and substantial agreement with both adverbs. Since we believe that the analysis of argumentative indicators requires expert annotators, we consider this result as a promising symptom of the guidelines' reliability. The analysis has shown that evidentemente and evidently function as indicators of premises-conclusion relations: they impose constraints on the position of the premises - which are intrasentential or adjacent - and they are compatible only with intrinsic argument schemes preferably of the causal type. More specifically, evidently and evidentemente are adverbs routinely used to signal the inference from observed effects to non-observable causes. The adverb evidently mainly selects inferences of thoughts, goals, values or beliefs from human observable behavior. To summarize, evidentemente and evidently are indicators both at the structural and at the inferential levels of argumentation. Therefore, they can be used as reliable features for both the automatic detection of argumentative components and argument schemes.

From the comparative analysis it has emerged that the Italian adverb, besides being more frequent, has more specific evidential functions than evidently. While evidentemente always functions as a marker of inferential evidentiality, evidently can also signal hearsay as an information source. Moreover, when not in sentence-initial position, evidently frequently conveys experiential inference where the perceptual sources of information are left vague, in line with its origin as a manner adverb. It, thus, seem that the indicative role played by evidentemente is more specific than that of evidently.

As to future work, we plan to extend this kind of analysis to the whole class of epistentials. One of the hypothesis we would like to test is whether certainty epistentials semantically related to perceptual evidence consistently work as indicators of intrinsic argument schemes. 


\section{Acknowledgements}

This study has been conducted thanks to the support of the Swiss National Foundation Early Post doc grant From semantics to argumentation mining in context: the role of evidential strategies as indicators of argumentative discourse relations (support: P2TIP1-165081).

\section{References}

[1] L.B. Anderson, Evidentials, paths of change, and mental maps: Typologically regular asymmetries, in: Evidentiality: The Linguistic Coding of Epistemology, W. Chafe and J. Nichols, eds, Ablex, Norwood, NJ, 1986, pp. 273-312.

[2] M. Becker, A. Palmer and A. Frank, Argumentative texts and clause types, in: Proceedings of the 3rd Workshop on Argument Mining, ACL, 2016, pp. 21-30.

[3] M. Becker, A. Palmer and A. Frank, Clause types and modality in argumentative microtexts, in: Proceedings of the COMMA Workshop "Foundations of the Language of Argumentation", 2016, pp. 1-9.

[4] F. Benamara, C. Cesarano, A. Picariello, D.R. Recupero and V.S. Subrahmanian, Sentiment analysis: Adjectives and adverbs are better than adjectives alone, in: ICWSM, 2007.

[5] J. Blochowiak, A theoretical approach to the quest for understanding: semantics and pragmatics of 'why's and 'because's, $\mathrm{PhD}$ thesis, University of Geneva, 2014.

[6] C. Clark, Evidence of evidentiality in the quality press 1993 and 2005, Corpora 5(2) (2010), 139-160. doi:10.3366/cor. 2010.0103.

[7] M.T. Faller, Semantics and pragmatics of evidentials in Cuzco Quechua, PhD thesis, Stanford University, 2002.

[8] J.B. Freeman, Dialectics and the Macrostructure of Arguments: A Theory of Argument Structure, Vol. 10, Walter de Gruyter, 1991. doi:10.1515/9783110875843.

[9] J.B. Freeman, What types of statements are there?, Argumentation 14(2) (2000), 135-157. doi:10.1023/A: 1007846431353.

[10] J.B. Freeman, Acceptable Premises: An Epistemic Approach to an Informal Logic Problem, Cambridge University Press, 2004. doi:10.1017/CBO9780511610301.

[11] J.B. Freeman, Argument Structure: Representation and Theory, Vol. 18, Springer Science \& Business Media, 2011. doi:10. 1007/978-94-007-0357-5.

[12] G. Gauthier, La structure et les fondements de l'argumentation editoriale, Les Cahiers du journalisme 17 (2007), 322-342.

[13] M. Halliday, Systemic Functional Linguistics, Hodder Education Publisher, New York, 1985.

[14] H. Haverkate, A speech act analysis of irony, Journal of Pragmatics 14(1) (1990), 77-109. doi:10.1016/03782166(90)90065-L.

[15] J. Lawrence and C. Reed, Argument mining using argumentation scheme structures, in: Computational Models of Argument: Proceedings of COMMA 2016, Frontiers in Artificial Intelligence and Applications, Vol. 287, IOS Press, 2016, pp. 379-390.

[16] J. Miecznikowski, Construction types and argumentative functions of possibility modals: Evidence from Italian, in: Proceedings of the 7th Conference of the International Society for the Study of Argumentation, 2011, pp. 1284-1297.

[17] J. Miecznikowski and E. Musi, Verbs of appearance and argument schemes: Italian sembrare as an argumentative indicator, in: Reflections on Theoretical Issues in Argumentation Theory, Springer, 2015, pp. 259-278.

[18] J. Moeschler, Argumentation and connectives, in: Interdisciplinary Studies in Pragmatics, Culture and Society, Springer, 2016, pp. 653-675.

[19] E. Musi, Evidential modals at the semantic-argumentative interface: Appearance verbs as indicators of defeasible argumentation, Informal Logic 34(4) (2014), 417-442. doi:10.22329/il.v34i4.4107.

[20] E. Musi, Dalle apparenze alle inferenze: i verbi sembrare e apparire come indicatori argomentativi, $\mathrm{PhD}$ thesis, Thèse de doctorat, Università della Svizzera italiana, 2015.

[21] E. Musi, Strategies of objectification in opinion articles: The case of evidentials, in: Proceedings of the 11th OSSA Proceedings of the 11th OSSA Conference "Argumentation, Objectivity and Bias", 2016.

[22] E. Musi, D. Ghosh and S. Muresan, Towards feasible guidelines for the annotation of argument schemes, in: Proceedings of the 3rd Workshop on Argument Mining, ACL, 2016, pp. 82-93.

[23] E. Musi and A. Rocci, Obviously epistentials are argumentative indicators: Evidence from an Italian and English corpus of newspaper articles, in: Proceedings of the COMMA Workshop "Foundations of the Language of Argumentation", 2016, pp. 48-55.

[24] J. Nuyts, Subjectivity as an evidential dimension in epistemic modal expressions, Journal of pragmatics 33(3) (2001), 383-400. doi:10.1016/S0378-2166(00)00009-6. 
[25] C. Perelman, The new rhetoric, in: Pragmatics of Natural Languages, Springer, 1971, pp. 145-149.

[26] R. Pinto, Argument, Inference and Dialectic: Collected Papers on Informal Logic, Vol. 4, Springer Science \& Business Media, 2001. doi:10.1007/978-94-017-0783-1.

[27] E. Rigotti and S.G. Morasso, Comparing the argumentum model of topics to other contemporary approaches to argument schemes: The procedural and material components, Argumentation 24(4) (2010), 489-512. doi:10.1007/s10503010-9190-7.

[28] A. Rocci, Modality and its conversational backgrounds in the reconstruction of argumentation, Argumentation 22(2) (2008), 165-189. doi:10.1007/s10503-007-9065-8.

[29] A. Rocci, Modality and argumentative discourse relations: A study of the Italian necessity modal dovere, Journal of Pragmatics 44(15) (2012), 2129-2149. doi:10.1016/j.pragma.2012.09.007.

[30] A. Rocci, Modal conversational backgrounds and evidential bases in predictions: The view from the Italian modals, in: Time: Language, Cognition and Reality, Oxford University Press, 2013, pp. 128-156.

[31] A. Rocci, Modality in Argumentation, Springer Science \& Business Media, 2017.

[32] A.-M. Simon-Vandenbergen and K. Aijmer, The Semantic Field of Modal Certainty: A Corpus-Based Study of English Adverbs, Vol. 56, Walter de Gruyter, 2007. doi:10.1515/9783110198928.

[33] S. Somasundaran, J. Ruppenhofer and J. Wiebe, Detecting arguing and sentiment in meetings, in: Proceedings of the SIGdial Workshop on Discourse and Dialogue, Vol. 6, 2007.

[34] C. Stab and I. Gurevych, Annotating argument components and relations in persuasive essays, in: COLING, 2014, pp. $1501-1510$.

[35] C. Stab and I. Gurevych, Identifying argumentative discourse structures in persuasive essays, in: EMNLP, 2014, pp. 4656.

[36] R. Swanson, B. Ecker and M. Walker, Argument mining: Extracting arguments from online dialogue, in: Proceedings of the 16th Annual Meeting of the Special Interest Group on Discourse and Dialogue, 2015, pp. 217-226.

[37] M. Taboada, J. Brooke, M. Tofiloski, K. Voll and M. Stede, Lexicon-based methods for sentiment analysis, Computational Linguistics 37(2) (2011), 267-307. doi:10.1162/COLI_a_00049.

[38] E.C. Traugott, On the rise of epistemic meanings in English: An example of subjectification in semantic change, Language 65 (1989), 31-55.

[39] A. Tseronis, From connectives to argumentative markers: A quest for markers of argumentative moves and of related aspects of argumentative discourse, Argumentation 25(4) (2011), 427-447. doi:10.1007/s10503-011-9215-x.

[40] A. Tseronis et al., Qualifying Standpoints. Stance Adverbs as a Presentational Device for Managing the Burden of Proof, LOT, Netherlands Graduate School of Linguistics, Utrecht, 2009.

[41] F.H. Van Eemeren and R. Grootendorst, A Systematic Theory of Argumentation: The Pragma-Dialectical Approach, Vol. 14, Cambridge University Press, 2004.

[42] F.H. Van Eemeren, P. Houtlosser and A.S. Henkemans, Argumentative Indicators in Discourse: A Pragma-Dialectical Study, Vol. 12, Springer Science \& Business Media, 2007.

[43] D. Walton, C. Reed and F. Macagno, Argumentation Schemes, Cambridge University Press, 2008. doi:10.1017/ CBO9780511802034.

[44] P.R. White, Dialogue and inter-subjectivity: Reinterpreting the semantics of modality and hedging, in: Dialogue Analysis VII: Working with Dialogue, Max Niemeyer Verlag, 2000, pp. 67-80.

[45] P.R. White, Beyond modality and hedging: A dialogic view of the language of intersubjective stance, Text 23(2) (2003), 259-284. doi:10.1515/text.2003.011.

[46] T. Willett, A cross-linguistic survey of the grammaticization of evidentiality, Studies in Language. International Journal sponsored by the Foundation "Foundations of Language" 12(1) (1988), 51-97.

[47] A. Wyner, J. Schneider, K. Atkinson and T.J. Bench-Capon, Semi-automated argumentative analysis of online product reviews, in: Computational Models of Argument: Proceedings of COMMA 2012, Frontiers in Artificial Intelligence and Applications, Vol. 245, IOS Press, 2012, pp. 43-50. 